\title{
MÉTHODE SURE ET RAPIDE D'APPRÉCIATION DES LAITS DESTINÉS A LA PASTEURISATION
}

\author{
par E. VAILLANT \\ Ingénieur agronome.
}

Au cours d'une recherche de documentation dans Le Lait (année 1923), mes yeux s'arrêtèrent sur l'analyse d'un article de W. RIEDEL dans Molkerei Zeitung Hildesheim, du 20 mars 1922, intitulé "Détermination de la valeur du lait " (voir Le Lait, 1923, pages 127-128) dont j'extrais les lignes suivantes :

"Il y a lieu de distinguer trois causes principales de coagulation du lait : fermentation lactique pure ; fermentation chymasique pure (ferment $1 \mathrm{ab})$; fermentation due à ces deux eauses simultanément.

« En 1913, Morres a proposé un procédé de titration (qui passa complètement inaperçu) qui mériterait d'être étudié et vérifié, car il serait de nature à rendre des services inappréciables pour l'appréciation du lait de consommation. "

Suit un résumé rapidé et sommaire de la thèse de MorRes, puis l'article se termine par ces mots :

"Si cette méthode était reconnue valable, elle dispenserait de l'essai au lacto-fermentateur, de la détermination de la réductase et de la numération des germes. Elle permettrait de suivre l'action de la présure surtout dans la fabrication des fromages à pâte molle. C'est pour cela qu'il y aurait un très grand intérêt à ce que ce procédé soit expérimenté en grand et perfectionné si cela est possible. *

Songeant aux difficultés que rencontrent notamment les industriels se livrant à la pasteurisation du lait lorsqu'il s'agit de trier consciencieusement les produits qu'ils reçoivent ainsi qu'à l'anxiété dans laquelle ils se trouvent lorsqu'ils constatent, en été notamment, l'état de fermentation des laits livrés, j'ai essayé de suivre les conseils de RIEDEL et d'étudier un mode pratique de titrage, reposant sur les principes ébauchés par Morres, adapté aux méthodes modernes de pasteurisation et susceptible de rendre à ces industriels de gros services.

J'ai tenu, avant tout, à concevoir une méthode rapide, utilisant un matériel restreint, bon marché, connu de tous et pouvant être manipulé par les plus inexpérimentés.

J'espère y être parvenu et serais particulièrement heureux que les expérimentateurs qui l'adopteront me fassent connaître les avantages ou les inconvénients qu'ils ont pu constater et veuillent bien me poser toutes questions et objections capables de m'aider à perfectionner cette méthode nouvelle ou à la modifier si nécessaire. Je leur en exprime à l'avance toute ma gratitude. 


\section{AVANT-PROPOS}

Il est essentiel, dans les usines de pasteurisation, d'écarter, en tout premier lieu, les lots de lait susceptibles de coaguler durant le traitement et d'éviter ainsi de graves désordres dans la marehe du travail et des ennuis non moins graves concernant la qualité et la bonne tenue des produits livrés à la consommation.

Pour exécuter ce premier triage, on se contente généralement d'effectuer un dosage de l'acidité, soit par un titrage selon la méthode de Dornic, soit par des réactions colorées diverses.

La question ne nous paraît pas envisagée sous son vrai jour et nous estimons qu'une telle pratique est vraiment trop incomplète, donc susceptible de nombreuses défaillances.

On nous objectera, il est vrai, que les usines possèdent d'autres méthodes plus scientifiques, plus précises, comme l'épreuve au lactofermentateur, l'épreuve de la catalase et de la réductase, la numération des germes, etc., qui leur permettent de suivre, en toute sécurité, les laits qu'on leur fournit.

Mais ce sont là des méthodes exigeant des manipulations assez délicates et qui ne donnent le renseignemènt qu'on en attend qu'au bout de plusieurs heures, voire même de plusieurs jours.

Nous estimons, pour notre part, qu'il est indispensable de les appliquer sans défaillance dans toutes les usines de pasteurisation et déplorons même qu'elles n'y soient pas rendues obligatoires mais elles ne peuvent nullement convenir pour le premier triage des laits à l'arrivée, qui ne précède que d'un temps très court le traitement de ces laits.

La méthode que nous voulons essayer de proposer ici vise particulièrement ce premier triage dont l'importance n'échappe à personne et c'est pourquoi nous avons voulu tenter de le rendre aussi rapide et aussi efficace que possible, toutes proportions gardées.

\section{CONSIDÉRATIONS GENERALES}

Pour comprendre le but que nous poursuivons, il faut avant tout bien connaître les causes de coagulation spontanée du lait.

La coagulation spontanée du lait est due à deux facteurs bien distincts, mais qui agissent ensemble et conjointement dans les laits de mélange tels que les reçoivent les laiteries.

Tout lait pur, sain, récolté avec tous les soins d'hygiène voulus, ne contenant aucun microorganisme nuisible, possède une acidité originelle qui varie entre $16^{\circ} \mathrm{D}$. et $20^{\circ} \mathrm{D}$, cad. entre $1 \mathrm{gr}$. 6 et $2 \mathrm{gr}$. d'acidité exprimée en acide lactique par litre.

Hors de ces limites deux cas peuvent se présenter :

ou bien l'acidité constatée est inférieure à $16^{\circ} \mathrm{D}$. : on se trouve en présence d'un lait " malade ", d'un lait dit "alcalin ", anormal 
et toujours dangereux parce qu'il provient d'une mamelle gravement atteinte. Un tel lait doit être éliminé d'emblée de toute usine. Il y aura intérêt à analyser ce lait au laboratoire afin de déceler les causes de cette anomalie et d'appeler aussitôt l'attention du producteur ;

ou bien l'acidité du lait est supérieure à $20^{\circ} \mathrm{D}$, et dans ce cas on se trouve en présence d'un lait où pullulent des ferments lactiques. qui ne vont pas tarder à provoquer sa coagulation spontanée. Ce lait est beaucoup moins dangereux que le précédent, mais, au point de vue industriel, toutes précautions doivent être prises pour en accepter ou en refuser l'entrée à l'usine.

C'est sur la discrimination des laits à accepter ou à refuser reposant uniquement sur l'examen de leur acidité que repose la méthode de triage la plus répandue dans les usines.

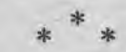

Mais en dehors du facteur "acidité ) résultant de la transformation du lactose en acide lactique par les ferments qui se développent dans le lait, il existe un autre facteur de eoagulation auquel on ne prête pas assez d'attention.

Ce facteur, que nous appellerons le "facteur lab", résulte de l'action coagulante d'une diastase, appelée lab ou présure, et qui est sécrétée par un grand nombre de microorganismes qui infectent généralement les laits de mélange courants.

Cette coagulation est totalement indépendante de celle provoquée par l'excès d'acide lactique, mais, en pratique, dans les laits apportés aux laiteries, ces deux actions se superposent et se complètent.

Ce qui veut dire qu'un lait ne eontenant que des ferments lactiques acidifiants exigera pour coaguler une acidité assez élevée ; par contre, s'il contient en outre du lab, cette coagulation spontanée aura lieu à un degré d'acidité moindre, l'action du lab venant s'ajouter à celle de l'acide lactique.

Mais, empressons-nous de dire que l'action du lab est très sournoise : presque nulle à la température ordinaire, elle se manifeste avec violence lorsque le lait est chauffé.

C'est ce qui nous a fait dire que la méthode de triage par dosage de l'acidité pouvait présenter de nombreuses défaillances qu'il est indispensable de réduire aussi complètement que possible.

\section{PRINCIPE DE LA MÉTHODE}

Ceci posé, essayons de voir comment il sera possible de connaître rapidement et avec une sûreté suffisante, l'action de ces deux facteurs " acidité " et "lab ", et d'estimer, par des chiffres, cette action. 
Nous savons qu'un lait pur, sain, soumis à la seule fermentation lactique pure, coagule, à la température ordinaire, lorsque l'acidité atteint de $70^{\circ} \mathrm{D}$. à $75^{\circ} \mathrm{D}$.

Par contre, si on chauffe ce lait en voie d'acidification lactique, la coagulation spontanée se fait beaucoup plus prématurément. A $40^{\circ} \mathrm{C}$. la coagulation se produit avec une acidité de $50^{\circ} \mathrm{D}$., à $65^{\circ} \mathrm{C}$. avee une acidité de $36^{\circ} \mathrm{D}$, à l'ébullition avec une acidité de $28^{\circ} \mathrm{D}$. seulement.

Done le "point de coagulation" dû à la seule acidité, diminue au fur et à mesure que la température augmente. A noter que l'acidité naturelle du lait frais est sans influence sur la bonne tenue du lait à toutes les températures normalement atteintes, soit pour la pasteurisation, soit pour la stérilisation.

$$
*^{*} *
$$

D'autre part, si on examine l'action du lab, prise isolément on constate des phénomènes très différents des précédents, lorsque la température du lait varie.

Comme toutes les diastases, le lab a une action relativement nulle à la température ordinaire $\left(15^{\circ} \mathrm{C}.\right)$, elle s'accroît rapidement pour atteindre son maximum vers $40^{\circ} \mathrm{C}$., puis baisse très vite pour cesser vers $60^{\circ} \mathrm{C}$., température qui le détruit.

$C^{\prime}$ 'est donc à une température de $40^{\circ}$ C. qu'il sera possible d'apprécier en toute certitude l'action du lab sur le lait.

$$
\text { ** * }
$$

Nous voici done en possession de trois renseignements importants qui vont nous permettre de passer à l'élaboration pratique de la méthode proposée, à savoir :

$1^{\circ}$ Un lait pur, sain et parfaitement propre, a une acidité naturelle allant de $16^{\circ} \mathrm{D}$. ̀̀ $20^{\circ} \mathrm{D}$, soit en moyenne à $18^{\circ} \mathrm{D}$.

$2^{\circ}$ Une fermentation lactique pure provoque dans le lait une acidité croissante qui peut faire coaguler le lait, à la température ordinaire lorsqu'elle atteint $70^{\circ} \mathrm{D}$. à $75^{\circ} \mathrm{D}$., et à la température de $40^{\circ} \mathrm{C}$. Jorsqu'elle atteint $50^{\circ} \mathrm{D}$.

$3^{\circ}$ L'action coagulante du lab sécrété par les ferments est nulle à $15^{\circ} \mathrm{C}$, passe par un maximum à $40^{\circ} \mathrm{C}$. et décroît ensuite.

Comme la méthode doit s'appliquer à des laits destinés à être pasteurisés, c'est-à-dire chauffés, nous nous placerons à la température optima pour les expériences, température qui sera certainement franchie lors du traitement de ces laits. Nous adopterons done la température de $40^{\circ} \mathrm{C}$.

A $40^{\circ}$ C., la fermentation lactique fait coaguler le lait dès que 
l'acidité atteint $50^{\circ} \mathrm{D}$, c'est-à-dire $32^{\circ} \mathrm{D}$. de plus que san acidité naturelle moyenne (18 D.).

A $40^{\circ}$ C., l'action du lab passe par son maximum.

$$
\text { *** }
$$

\section{LE DOSAGE}

Le dosage comprend trois opérations très simples, exécutées avec deux acidimètres Dornic.

L'un des acidimètres contient la solution de soude, habituellement utilisée pour le titrage du degré Dornic d'acidité.

L'autre contient une solution d'acide sulfurique titrée de telle façon qu'elle neutralise exactement, volume pour volume, la solution de soude précédente.

Il sera nécessaire de posséder un récipient dans lequel on entretiendra de l'eau à la température de $40^{\circ} \mathrm{C}$.

Pour chaque lait à analyser on prélèvera, dans des tubes à essai, trois échantillons de $10 \mathrm{~cm}^{3}$ exactement. On plongera l'un de ces tubes seulement dans le bain-marie à $40^{\circ} \mathrm{C}$.

\section{A. Dosage de l'acidité à la température ordinaire}

On effectue, sur un des tubes, non chauffé, le titrage d'acidité habituel, selon la méthode Dornic, à l'aide de la solution de soude.

Ajouter au lait 5 gouttes de solution de phtaléine; faire couler doucement la soude à l'aide de la burette graduée en agitant de temps en temps jusqu'à ce que le lait prenne une teinte rose persistante.

La division où s'arrête le liquide dans la burette donne immédiatement le degré d'acidité.

Moins de 16 divisions : lait malade à rejeter.

De 16 à 20 divisions : lait normal.

Plus de 20 divisions : lait subissant une fermentation lactique.

A surveiller.

\section{B. Détermination du degré de coagulation à froid}

Ce dosage s'effectue sur le second tube non chauffé.

On utilise cette fois l'acidimètre contenant la solution d'acide sulfurique titrée. Ne pas mettre de phtaléine dans le lait.

Faire tomber goutte à goutte la solution acide contenue dans la burette graduée en agitant après chaque addition pour dissoudre les petits flocons formés par le lait au contact de l'acide. Continuer jusqu'à coagulation complète. Ce point est très facile à observer.

La division où s'arrête le liquide dans la burette donne immédiatement le degré de coagulation à froid. 


\section{Détermination du degré de coagulation à $40^{\circ} \mathrm{C}$.}

Opérer exactement comme ci-dessus (essai B.), mais eette fois, sur le tube plongé dans le bain-marie à $40^{\circ} \mathrm{C}$.

N.-B. - Ces deux déterminations du degré de coagulation consistent tout bonnement à provoquer artificiellement et instantanément la coagulation du lait, comme elle aurait pu se faire à la longue par l'acide lactique, en substituant à cet acide lactique un acide titré équivalent dont l'emploi immédiat permet de mesurer la quantité d'acide lactique qui aurait été encore nécessaire pour coaguler ce lait.

\section{INTERPRÉTATION DES RÉSULTATS}

Pour un lait déterminé, nous sommes donc en possession des trois résultats suivants :

Le degré d'acidité Dornic, obtenu à la température ordinaire. naire.

Le degré de coagulation à froid,obtenu à la température ordi-

Le degré de coagulation à $40^{\circ} C$, obtenu sur lait chauffé à $40^{\circ} \mathrm{C}$.

Le degré d'acidité nous permet immédiatement de connaître les laits anormaux ou malades et les laits en voie d'acidification.

Examinons maintenant le parti que nous pouvons tirer des degrés de coagulation à froid et à $40^{\circ} \mathrm{C}$.

Nous savons qu'un lait idéal a une acidité naturelle de $18^{\circ} \mathrm{D}$. en moyenne, et qu'une fermentation lactique pure le fait coaguler à $40^{\circ} \mathrm{C}$. lorsque l'acidité atteint $50^{\circ} \mathrm{D}$. C'est-à-dire lorsque l'acidité s'est accrue de $50-18=32^{\circ} \mathrm{D}$.

On peut done dire qu'à $40^{\circ} \mathrm{C}$., le degré de coagulation maximum d'un lait idéalement pur sera de $32^{\circ}(1)$ et que le degré minimum sera $0^{\circ}$ pour un lait coagulant spontanément dans le tube placé au bain-marie.

Mais on ne pasteurise pas à $40^{\circ} \mathrm{C}$., et chacun sait qu'un lait acidifié peut ne pas coaguler à $40^{\circ} \mathrm{C}$. et cependant cailler totalement lorsqu'il atteint la température de pasteurisation.

Les limites ei-dessus doivent donc être modifiées pour des températures supérieures à $40^{\circ} \mathrm{C}$., soit :

$65^{\circ}$ pour la pasteurisation basse ;

$75^{\circ}$ pour les pasteurisations instantanées et notamment la stassanisation.

Or, nous savons $q u^{\prime} \grave{a} 65^{\circ} \mathrm{C}$. un lait coagule avec une acidité

(1) Pour éviter toute confusion dans la lecture des degrés, ceux-ci seront suivis de la lettre $\mathrm{C}$ pour les températures et de la lettre D pour l'acidité ; les degrés de coagulation ne seront suivis d'aucune lettre. 
de $36^{\circ} \mathrm{D}$. et à $75^{\circ} \mathrm{C}$. avec une acidité de $32^{\circ} \mathrm{D}$, ce qui veut dire que l'écart entre l'acidité naturelle du lait pur (18 $18^{\circ}$ D.) et celle de coagulation à ces températures de pasteurisation est :

Pasteurisation basse : $36-18=18^{\circ}$ de coagulation.

Stassanisation : $32-18=14^{\circ}$ de coagulation.

Si le maximum que peut atteindre le degré de coagulation à toutes les températures courantes demeure immuablement fixé à $32^{\circ}$ pour un lait idéalement pur, il n'en est pas de même du minimum qui, de $0^{\circ}$ à la température de $40^{\circ} \mathrm{C}$. devient :

lation.

Pour $65^{\circ} \mathrm{C}$. (pasteurisation basse) : $32-18=14^{\circ}$ de coagu-

Pour $75^{\circ} \mathrm{C}$. (stassanisation) : $32-14=18^{\circ}$ de coagulation.

En résumé, nous retiendrons donc qu'un lait exclusivement soumis à une fermentation lactique pure, ne coagulera pas à la température de pasteurisation si son degré de coagulation à $40^{\circ} \mathrm{C}$. est compris dans les limites suivantes :

Pasteurisation basse $\left(65^{\circ} \mathrm{C}\right.$. $)$ : entre $32^{\circ}$ et $14^{\circ}$.

Stassanisation ( $75^{\circ}$ C.) : entre $32^{\circ}$ et $18^{\circ}$.

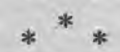

Nous avons systématiquement écarté, jusqu'ici, de nos considérations l'action simultanée du lab qui peut hâter la coagulation des laits chauffés et par conséquent fausser complètement les données que nous venons de poser.

Il s'agit done maintenant de déterminer cette action du lab, de l'exprimer en degrés que nous appellerons "degrés lab" et de fixer l'influence de ces degrés lab sur les limites que nous avons établies à la fin du paragraphe précédent.

Rappelons encore une fois que le lab n'agit pas à la température ordinaire, mais, par contre, que son action est maximum à $40^{\circ} \mathrm{C}$.

En conséquence, si un lait contient du lab, son degré de coagulation à $40^{\circ} \mathrm{C}$. sera inférieur au degré de coagulation du même lait pris à froid, et la différence entre ces deux degrés de coagulation correspondra évidemment à la seule action du lab.

C'est le chiffre exprimé par cette différence qui constitue ce que nous avons convenu d'appeler le "degré lab".

Prenons un exemple :

On obtient avec un lait les résultats suivants :

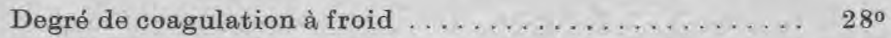

Degré de coagulation à $40^{\circ} \mathrm{C} . \ldots \ldots \ldots \ldots \ldots \ldots \ldots \ldots \ldots \ldots \ldots \ldots \ldots 1^{\circ}$

on en déduit que le degré lab est : $28-21=7^{\circ}$ lab.

Le degré lab s'ajoute à l'acidité pour hâter la coagulation et, par réciprocité, il tend à réduire le degré de coagulation à $40^{\circ} \mathrm{C}$. 
Il ne peut pas agir en abaissant la limite maxima fixée cidessus, puisque celle-ci correspond à un lait pur ne contenant ni ferments lactiques ni ferments lab; il ne peut done agir que sur la limite minima qu'il élève.

C'est ainsi que dans l'exemple ci-dessus, si nous avons affaire à la pasteurisation basse, le degré de coagulation à $40^{\circ}$ ne devra pas descendre au-dessous de $14+7=21^{\circ}$, et si nous avons affaire à la stassanisation, il ne devra pas être inférieur à $18+7=25^{\circ}$.

C'est done autour de cette limite minima corrigée que se jugera la valeur industrielle d'un lait, aussi lui avons-nous donné le nom de "degré d'utilisation du lait ".

Toutes les fois que le degré de coagulation à $40^{\circ} \mathrm{C}$. sera égal ou inférieur au degré d'utilisation le lait devra être systématiquement et irrémédiablement rejeté de la pasteurisation.

Notons enfin que le degré d'utilisation d'un lait est particulier à ce lait, puisque dans sa détermination rentre un facteur propre à ce lait qui est le degré lab.

Ces considérations, un peu longues, ont pour but de donner les justifications de la méthode proposée. En pratique, les industriels n'ont qu'à se conformer simplement aux indications qui vont suivre. Nous sommes convaincu que la sécurité absolue que leur procurera cette méthode attirera toute leur attention.

\section{RÉSUMÉ PRATIQUE A L'USAGE DES INDUSTRIELS}

\section{Matériel.}

2 acidimètres Dornic, dont l'un est rempli de solution titrée de soude, et l'autre de solution titrée d'acide sulfurique.

1 pipette de $10 \mathrm{~cm}^{3}$.

Des tubes à essai.

1 flacon compte-gouttes pour la solution de phtaléine.

1 bain-marie de forme quelconque, muni d'un thermomètre, dans lequel on maintiendra de l'eau à $40^{\circ} \mathrm{C}$.

\section{Réactifs.}

Faire préparer dans un laboratoire de chimie les solutions suivantes :

a) Solution titrée de soude. $9 \mathrm{~cm}^{3}$ de cette solution doivent neutraliser exactement $10 \mathrm{~cm}^{3}$ de solution titrée d'acide sulfurique déeinormale.

b) Solution titrée d'acide sulfurique. Cette solution doit neutraliser exactement, volume à volume, la solution de soude ci-dessus.

c) Solution de phtaléine. Dissoudre complètement par agitation 2 grammes de phtaléine du phénol dans $100 \mathrm{~cm}^{3}$ d'alcool à $68-70^{\circ}$. 


\section{Analyses,}

Bien mélanger le lait à analyser. A l'aide de la pipette, mettre exactement $10 \mathrm{~cm}^{3}$ de ce lait dans trois tubes à essai. L'un de ces tubes sera maintenu dans le bain-marie à $40^{\circ} \mathrm{C}$.

Pour la technique des analyses, voir plus haut, au paragraphe "Le dosage ".

Ces analyses terminées on est en possession des résultats suivants :

Le degré Dornic, ou degré d'acidité.

Le degré de coagulation à froid,

Le degré de coagulation à $40^{\circ} \mathrm{C}$. lation.

Le degré lab obtenu par différence des deux degrés de coagu-

\section{Interprétation des résultats.}

Si le degré d'acidité est inférieur à $16^{\circ}$ D., le lait provient de vaches dont le pis est sérieusement malade. Lait dangereux et impropre à tous usages. A rejeter.

Si le degré d'acidité est compris entre $16^{\circ} \mathrm{D}$, et $20^{\circ} \mathrm{D}$. et que le degré lab est voisin de zéro, le lait est de toute première qualité.

Si le degré d'acidité dépasse $20^{\circ} \mathrm{D}$. à $22^{\circ} \mathrm{D}$, ou si le degré lab s'élève, il y a lieu de comparer les résultats enregistrés avec les chiffres limites fixés pour chaque mode de pasteurisation.

a) Pasteurisation basse. - Déterminer le degré d'utilisation du lait en ajoutant le chiffre fixe 14 au degré lab.

Si le degré de coagulation à $40^{\circ} \mathrm{C}$. est supérieur au degré d'utilisation ci-dessus, le lait peut être pasteurisé sans aucune crainte.

Si le degré de coagulation à $40^{\circ} \mathrm{C}$. est égal ou inférieur au degré d'utilisation ci-dessus, le lait ne peut supporter la pasteurisation et doit être envoyé à l'écrémeuse immédiatement.

b) Stassanisation. - Déterminer le degré d'utilisation du lait en ajoutant le chiffre fixe 18 au degré lab.

Pour le reste, comme ci-dessus.

\section{QUELQUES EXEMPLES \\ Exemple 1}

Degré d'acidité

$20^{3}$ D.

Degré de coagulation à froid $\ldots \ldots \ldots \ldots \ldots \ldots \ldots 0^{\circ}$

Degré de coagulation à $40^{\circ} \mathrm{C} \ldots \ldots \ldots \ldots \ldots \ldots \ldots . \ldots 29^{\circ}$

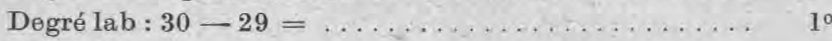

Lait excellent à tous les points de vue.

\section{Exemple 2}

Degré d'acidité ........................ $21^{\circ} \mathrm{D}$

Degré de coagulation à froid $\ldots \ldots \ldots \ldots \ldots \ldots \ldots 28^{\circ}$ 
Degré de eoagulation à $40^{\circ} \mathrm{C} \ldots \ldots \ldots \ldots \ldots \ldots \ldots \ldots \ldots \ldots \ldots . \ldots 22^{\circ}$

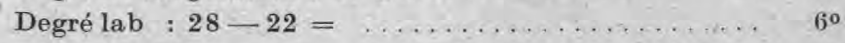

Degré d'utilisation :

Pasteurisation basse : $6+14=20^{\circ}$.

Stassanisation : $6+18=24^{\circ}$.

Ce lait peut encore supporter la pasteurisation basse à $63^{\circ}-65^{\circ}$, mais ne supporterait pas la stassanisation. En effet, son degré de coagulation à $40^{\circ} \mathrm{C}$ qui est $22^{\circ}$ est supérieur à $20^{\circ}$ et inférieur à $24^{\circ}$.

\section{Exemple 3}

Degré d'acidité ....................... $23^{\circ} \mathrm{D}$.

Degré de coagulation à froid $\ldots \ldots \ldots \ldots \ldots \ldots \ldots .25^{\circ}$

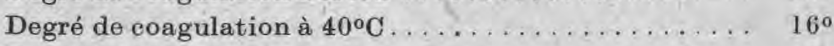

Degré lab : $25-16=\ldots \ldots \ldots \ldots \ldots \ldots \ldots \ldots \ldots \ldots \ldots \ldots \ldots$

Degré d'utilisation :

Pasteurisation basse : $9+14=23^{\circ}$.

Stassanisation : $9+18=27^{\circ}$.

Le degré de coagulation à $40^{\circ} \mathrm{C}$ étant notablement inférieur à ces degrés d'utilisation, le lait est à diriger vers l'écrémeuse.

Le degré d'acidité et le degré lab, tous deux très élevés, indiquent d'ailleurs que ee lait est en pleine fermentation.

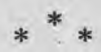

Cette méthode exige évidemment trois essais, mais elle offre pour l'industriel l'énorme avantage de le renseigner avec certitude et en quelques minutes sur la destination qu'il doit donner aux divers laits qu'il reçoit, renseignement essentiel qu'il lui serait impossible de connaitre avant plusieurs heures par l'emploi du lacto-fermentateur, de l'épreuve de la réductase, de la numération des germes, etc.

Néanmoins, nous maintenons qu'il est absolument indispensable, dans les usines sérieuses et bien menées, de pratiquer régulièrement ces essais plus précis et plus scientifiques dans le but d'étudier, après coup, sur les échantillons retenus et étiquetés les livraisons anormales de certains fournisseurs, de déceler les causes de ces anomalies (maladies ou malpropreté), de prendre toutes dispositions pour les faire disparaître au plus tôt et d'appliquer, si nécessaire, les sanctions qui s'imposent.

$$
\text { *** }
$$

La méthode qui vient d'être exposée peut rendre également de très grands services aux industriels fromagers. On sait, en effet, l'importance capitale de la qualité des laits en fromagerie et son influence sur la technique du travail et sur la valeur des produits fabriqués. 